\title{
Broadcast Capacity for Wireless Ad Hoc Networks
}

\author{
Xiang-Yang Li, Shao-Jie Tang, Xiao-Hua Xu \\ Dept. of Computer Science \\ Illinois Institute of Technology \\ Chicago, IL 60616, USA \\ \{xli, stang7, xxu23\}@iit.edu
}

\begin{abstract}
The capacity of a wireless network has been widely studied in the literature, including the capacity for unicast and the capacity for broadcast. In this paper, we studied the capacity of a wireless network for broadcast. Previous studies on broadcast capacity either assume that all links in the wireless network has the same channel capacity, or assume that the transmission ranges of a wireless node can be arbitrarily large. In this paper we derive analytical upper bounds and lower bounds on broadcast capacity of a wireless network when all nodes in the network has the same bounded transmission power $P$ and all nodes are placed in a square of side-length $a$. When the fixed data rate channel is used (each node can send $W$ bits/second to nodes within its transmission range if no interference happened), we prove that the broadcast capacity is $\Theta(W)$ under the physical interference model.

When the Gaussian channel capacity is used, we show that the total broadcast capacity is only $\Theta\left(\left(a \sqrt{\frac{\log n}{n}}\right)^{-\alpha}\right)$ when $a \sqrt{\frac{\log n}{n}} \rightarrow \infty$. When $a \sqrt{\frac{\log n}{n}} \rightarrow O(1)$, we show that the broadcast capacity is $\Theta(1)$. We also generalize our results to multicast capacity for physical interference model.
\end{abstract}

\section{Introduction}

In wireless ad hoc networks, wireless nodes may cooperate in routing each others' packets. Lack of a centralized control of the functionality and possible node mobility give rise to many challenging issues at the network, medium access and physical layers of a wireless ad hoc network. At the network layer, the main challenging problem is that of routing, which has to deal with time-varying network topology, possible power-constraints of wireless nodes, and the characteristics of the wireless channel (such as unstable, broadcast nature, fading and so on). The choice of medium access control is also restricted by the fact that the network topology is time-varying, and there is no centralized control. In the literature, a number of results have been proposed to use the TDMA, CDMA, and the dynamic assignment of frequency bands to improve the network throughput. Notice that for a mobile wireless network, random access appears to the current favorite protocols due to its simplicity and quick adaption to mobility and dynamic data rate by nodes. For a mobile wireless network, static FDMA is inefficient in dense networks, CDMA is very difficult to implement due to node mobility and the need for keeping track of spreading codes for nodes in the time-varying neighborhoods. Notice that TDMA has recently been proposed to improve the network throughput for some networks or partial of the networks [1], especially for static networks. At the physical layer an important issue is the power-control, which has been studied extensively in the literature. A careful selection of the transmission power of nodes will not only improve the nodal life, but also improve the spatial reuse of frequency and possibly improve the network throughput consequently.

In some applications, e.g., wireless sensor networks, we often need a rough estimation on the achievable throughput when we randomly deployed $n$ number of wireless nodes in a given region. The main purpose of this paper is to study the capacity of wireless networks when we choose the best protocols for all layers. We will study the capacity of a given wireless network where the nodes positions are given a priori, and how the capacity of wireless networks scale with the number of nodes in the networks (when given a fixed deployment region), or scale with the size of the deployment region (when given a fixed deployment density) for a various number of operations such as unicast, broadcast. Due to spatial separation, several wireless nodes can transmit simultaneously provided that these transmissions will not cause destructive wireless interferences to any of the transmissions. As in the literature, we will mainly consider two types of networks, arbitrary networks and random networks. In random networks, we assume that a set of $n$ 
wireless nodes are randomly distributed in a fixed region (such as a unit square by a proper scaling). In an arbitrary network, the node locations, destinations of the sources, and traffic demands are all arbitrary. All $n$ nodes are arbitrarily located in the deployment region.

For broadcast, each node $v_{i}$ wishes to send traffic at an arbitrary data rate $\lambda_{i} \geq 0$ to all nodes in the network. As always, we assume that the packets are sent from node to node in a multi-hop manner until they reach their final destinations. The packets could be buffered at intermediate nodes while awaiting for transmission. In this paper, we assume that the buffer is large enough so packets will not get dropped by intermediate nodes. We leave it as future work to study the scenario when the buffers of intermediate nodes are bounded by some values. In some results, we assume that every intermediate nodes have infinite buffer size. For most of the results presented here, the delay of the routing is not considered, i.e., the delay in the worst case could be arbitrarily large for some results.

In both arbitrary network or random networks, we assume that each node will choose a uniform transmission range or transmission power $P$. Two different channel models will be used in this paper.

1. The first channel model assumes a fixed data rate. We assume that each wireless node can transmit at $W$ bits/second over a common wireless channel. For presentation simplicity, we assume that there is only one channel in the wireless networks. We will see that it is immaterial to the results presented in this paper if the channel is broken up into several sub-channels of capacity $W_{1}, W_{2}, \cdots, W_{M}$ bits/second as long as we have $\sum_{i=1}^{M} W_{i}=W$. To describe when a transmission is received successfully by its intended receiver(s), we will allow two possible models for a successful onehop reception: protocol model and the physical model.

2. The second channel model is the Gaussian channel. The channel capacity from node $v_{i}$ to node $v_{j}$ is $B \cdot \log \left(1+\frac{P_{i} \cdot d_{i, j}^{-\alpha}}{B \cdot N_{0}+\sum_{k \in I} P_{k} \cdot d_{k, j}^{-\alpha}}\right)$. Here $B$ is the channel bandwidth and $d_{i, j}$ is the Euclidean distance $\left\|v_{i}-v_{j}\right\|$, $N_{0}>0$ is the background Gaussian noise, $I$ is the set of actively transmitting nodes when node $v_{i}$ is transmitting, $\alpha>2$ is the pass loss exponent, and $P_{k}$ is the transmitting power used by a node $v_{k}$.

Our Results: In this paper we derive analytical upper bounds and lower bounds on broadcast capacity of a wireless network when all nodes in the network has the same bounded transmission power $P$ and all nodes are placed in a square of side-length $a$. When the fixed data rate channel is used (each node can send $W$ bits/second to nodes within its transmission range if no interference happened), we prove that the broadcast capacity is $\Theta(W)$ under the physical interference model. This is the first result that studies the broadcast capacity for arbitrary networks using physical interference model.

When the Gaussian channel capacity is used, we show that the total broadcast capacity is only $\Theta\left(\left(a \sqrt{\frac{\log n}{n}}\right)^{-\alpha}\right)$ when $a \sqrt{\frac{\log n}{n}} \rightarrow \infty$. Our results imply the result for broadcast in random networks in [17] when $a=\sqrt{n}$, the broadcast capacity is $\Theta\left((\log n)^{-\alpha / 2}\right)$. When $a \sqrt{\frac{\log n}{n}} \rightarrow$ $O(1)$, we show that the broadcast capacity is $\Theta(1)$. This implies that the broadcast capacity is $\Theta(1)$ when all nodes' transmission power $P$ can only support the communication to nodes within a constant meters, in which case, the deployment region $a$ is at most $O\left(\sqrt{\frac{n}{\log n}}\right)$.

We then generalize our results to multicast when there are $n_{s}$ multicast sessions and each multicast source node will send data to $k-1$ nodes (randomly selected among $n-1$ other nodes) at data rate $\lambda_{i}$. We show that the aggregated multicast capacity of $n_{s}$ random multicasts is

$$
\Lambda_{k}(n)= \begin{cases}\Theta\left(\sqrt{\frac{n}{\log n}} \cdot \frac{W}{\sqrt{k}}\right) & \text { when } k=O\left(\frac{n}{\log n}\right), \\ \Theta(W) & \text { when } k=\Omega\left(\frac{n}{\log n}\right)\end{cases}
$$

The rest of the paper is organized as follows. In Section 2 we discuss in detail the network model and the channel model used in this paper. In Section 3, we first review and study the broadcast capacity for fixed networks with constant link capacity under both protocol interference model and physical interference model. We show that the integrated broadcast capacity of all sources is $\Theta(W)$ regardless of the number of sources nodes. In Section 4, we then study the asymptotic broadcast capacity for random networks when all nodes have the uniform transmission Power $P$ under the Gaussian channel. We then generalize our studies to multicast in Section 5. We review the related results on network capacities in Section 6 and conclude the paper in Section 7 with discussion of some future works.

\section{Network Model}

In this paper, we assume that there is a set $V=$ $\left\{v_{1}, v_{2}, \cdots, v_{n}\right\}$ of $n$ communication terminals deployed in a region $\Omega$. We mainly focus the scenario when $\Omega$ is a square with side length $a$. When the fixed data-rate channel is used, we assume that every wireless node has a transmission range $r$ such that a node $u$ can successfully receive the signal sent by node $v$ if and only if $\|u-v\| \leq r$. The complete communication graph is a directed graph $G=(V, E)$, where $V=\left\{v_{1}, \ldots, v_{n}\right\}$ is the set of terminals and $E$ is the set of directed communication links.

We assume that the transmission range (or transmission power) of all nodes are fixed. To get large scale networks, we increase the node density (asymptotically same as the 
node degree when the transmission range is fixed) and the deployment area to increase the number of nodes in the network. We call this model the fixed-range model. This is different from the dense model [6] (with fixed deployment region) and extended model (with fixed nodal density).

Channel Models: In this paper, two different channel models will be studied. The first channel model assumes a fixed data rate. We assume that each wireless node can transmit at $W$ bits/second over a common wireless channel. For presentation simplicity, we assume that there is only one channel in the wireless networks. we further assume that the transmission range $r$ is fixed and thus normalized to one unit. Under this assumption, the deployment region $\Omega$ will be a function of $n$. To schedule two links at the same time slot, we must ensure that the schedule will avoid the interference. Two different types of interference have been studied in the literature, namely, primary interference and secondary interference. In this paper, we assume two different interference models for wireless networks with fixed data-rate channel.

1. The first interference model is protocol interference model. All nodes are assumed to have uniform interference range $R$. A node $v_{j}$ will not receive the data correctly from a sender $v_{i}$ correctly if node $v_{j}$ lies inside the interference region of another actively transmitting node $v_{k}$, i.e., $\left\|v_{j}-v_{k}\right\| \leq R$.

2. The second is the physical interference model. A node $v_{j}$ can correctly receive the data from a sender $v_{i}$ if and only if, for a given constant $\eta>0$, $\frac{P_{i} \cdot d_{i, j}^{-\alpha}}{B \cdot N_{0}+\sum_{k \in I} P_{k} \cdot d_{k, j}^{-\alpha}} \geq \eta$. Here $d_{k, j}$ is the Euclidean distance $\left\|v_{k}-v_{j}\right\|, N_{0}>0$ is the background Gaussian noise, $I$ is the set of actively transmitting nodes when node $v_{i}$ is transmitting, $\alpha>2$ is the pass loss exponent, and $P_{k}$ is the transmitting power used by a node $v_{k}$.

The second channel model that will be studied in this paper is Gaussian channel. Let $P_{i}$ denote the power used by node $i$ for transmission. Assume that the maximum power for transmission by each node is $P_{\max }$. Assume that the channel follows ambient Gaussian noise model with power spectral density of $N_{0} / 2$ and the signal attenuation of $d^{-\alpha}$ where $d$ is the distance between the source and the receiver node. When some common information is directly broadcast from a node $i$ to a set of receivers $\mathcal{R}$, capacity-achieving Gaussian channel codes are assumed to support the worst achievable data rate of all receivers, i.e.,

$$
r_{i}=\min _{j \in \mathcal{R}} B \cdot \log \left(1+\frac{P_{i} \cdot d_{i, j}^{-\alpha}}{B \cdot N_{0}+\sum_{k \in I} P_{k} \cdot d_{k, j}^{-\alpha}}\right),
$$

where $I$ is the set of nodes that are simultaneously transmitting with node $i$ using the same channel and $B$ is the bandwidth of the channel. Notice that this model of data rate is different from the model of data rate used to study the capacity for unicast previously, where we typically assumed a fixed data rate $W$ for the channel. This is also different from the physical model used in previous sections in which we assumed a minimum bound on SINR, while here we do not have such constraint. As in [17], it is further assumed that no cooperative relay strategy is used at the physical layer to improve the throughput. Based on the rate definition supported by a node $i$ to its set of downstream children nodes $\mathcal{R}$, every sender node needs to determine the set $\mathcal{R}$ of receivers it needs to reach and chooses a coding scheme and the corresponding transmission power such that the receiver node with the least SINR can also successfully decode the message. For most results studied for random networks, we will assume that all nodes in the network have the uniform transmission power $P$.

Problems Studied: In this paper, we will study the asymptotic broadcast capacity of both arbitrarily networks and random networks with both fixed data rate channel model and the Gaussian channel model. Assume that each node $v_{i}$ will send data to all other nodes $U_{i}=V-\left\{v_{i}\right\}$ with a data rate $\lambda_{i}$. Let $\lambda=\left(\lambda_{1}, \lambda_{2}, \cdots, \lambda_{n-1}, \lambda_{n}\right)$ be the rate vector of the broadcast data rate of all possible $n$ broadcast sessions.

When given a fixed network $G=(V, E)$, where the node positions of all nodes $V$, the set of receivers $U_{i}$ for each source node $v_{i}$, and the broadcast data rate $\lambda_{i}$ for each source node $v_{i}$ are all fixed, we first define what is a feasible rate vector $\lambda$ for the network $G$.

Definition 1 (Feasible Rate Vector) A broadcast rate vector $\lambda=\left(\lambda_{1}, \lambda_{2}, \cdots, \lambda_{n-1}, \lambda_{n}\right)$ bits/sec is feasible if there is a spatial and temporal scheme for scheduling transmissions such that by operating the network in a multi-hop fashion and buffering at intermediate nodes when awaiting transmission, every node $v_{i}$ can send $\lambda_{i}$ bits/sec average to its chosen destination nodes. That is, there is a $T<\infty$ such that in every time interval (with unit seconds) $[(i-1) \cdot T, i \cdot T]$, every node can send $T \cdot \lambda_{i}$ bits to its corresponding $k-1$ receivers.

The total throughput capacity of such feasible rate vector for broadcast is defined as $\Lambda(n)=\sum_{i=1}^{n} \lambda_{i}$. The average per node broadcast throughput capacity is defined as $\lambda(n)=\frac{\sum_{i=1}^{n} \lambda_{i}}{n}$.

Definition 2 (Feasible Throughput Capacity) A throughput $\lambda(n)$ bits/sec for each node is feasible for broadcast if there is a rate vector $\lambda=\left(\lambda_{1}, \lambda_{2}, \cdots, \lambda_{n-1}, \lambda_{n}\right)$ that is feasible and $\frac{\sum_{i=1}^{n} \lambda_{i}}{n}$ is $\lambda(n)$. Similarly, we say $\Lambda(n)=$ $\sum_{i=1}^{n} \lambda_{i}$ is a feasible broadcast throughput capacity.

Definition 3 (Capacity of Random Networks) The aggregated broadcast capacity of a class of random networks 
is of order $\Theta(g(n))$ bits/sec if there are deterministic constants $c, c^{\prime}\left(0<c<c^{\prime}<+\infty\right)$ such that

$$
\begin{aligned}
\lim _{n \rightarrow \infty} \operatorname{Pr}(\Lambda(n)=c g(n) \text { is feasible }) & =1 \\
\liminf _{n \rightarrow \infty} \boldsymbol{P r}\left(\Lambda(n)=c^{\prime} g(n) \text { is feasible }\right) & <1
\end{aligned}
$$

Obviously, for random networks, the broadcast capacity $\Lambda(n)$ and the broadcast capacity per node $\lambda(n)$ satisfy the relation: $\lambda(n)=\frac{\Lambda(n)}{n}$. Similarly, we can define multicast capacity for random networks.

\section{Broadcast Capacity for An Arbitrary Net- work}

Broadcast capacity of single-source of an arbitrary network has been studied in $[8,15]$. They essentially show that the broadcast capacity of a given network is $\Theta(W)$ for single source broadcast. In this paper, we prove that the achievable integrated broadcast capacity is still only $\Theta(W)$ if each of an arbitrary subset of the $n$ nodes will serve as source node and different source nodes may have different data rate.

\subsection{Protocol Interference Model}

We first study the aggregated broadcast capacity for an arbitrary network when protocol interference model is used.

Theorem 1 Assume that the channel capacity is $W$ bits/sec. Under the protocol interference mode, the aggregated broadcast capacity $\Lambda(n)$ of an arbitrary wireless network is $\Theta(W)$.

Proof: If there is only one source node $s$ for broadcast, it is easy to show that the upper bound of broadcast is at most $W$ since the number of bits that come out of the source node is at most $W \cdot T$ in time $T$ seconds. Thus, the capacity is bounded from above by $W$. We show that $\Theta(W)$ is achievable as follows. Given a network $G$, we first create a connected dominating set (CDS) where the source node $s$ is part of the CDS. The method we used to create CDS could be any method that guarantees that each dominatee has at most a constant number of dominators in its one-hop neighborhood. The CDS constructed using those methods has the following nice property: for each node $v$ in the CDS, the number of communication neighbors of $v$ in CDS is bounded from above by a constant, say $c$. Assume that the interference range of every node is a constant $(1+\Delta)$ times of the communication range $r$. Notice that in practice, $\Delta$ typically is around 1 . Let $H$ be the interference graph constructed for CDS: the nodes of $H$ are nodes from the constructed CDS; two nodes $u$ and $v$ from CDS are connected in the interference-graph $H$ only if there is a node $w \in V^{4}$ such that $\|u-w\| \leq(1+\Delta) r$ and $\|v-w\| \leq r$. Then it is easy to show that the graph $H$ also has degree at most $c(2+\Delta)^{2}$. Consequently, we can color $H$ using at most $1+c(2+\Delta)^{2}$ colors. Let $t(v) \in\left[1,1+c(2+\Delta)^{2}\right]$ be the color assigned to node $v$ from the CDS. In other words, we can schedule the transmissions of all nodes in the CDS without causing any interference as follows: node $v$ only transmits at time $t(v)+i \cdot T$ where $T=1+c(2+\Delta)^{2}$. Then we can perform broadcast based on the constructed CDS as follows: a node $v$ in the CDS is scheduled to relay the data from its parent node at time $t(v)+i \cdot T$. Thus, the achieved data rate using such broadcast is $\frac{W}{T}=\Theta(W)$. Consequently, the achievable broadcast capacity is $\Theta(W)$ (where the lower bound and the upper bound matches).

When there are multiple sources in the network, it is not straightforward that the total broadcast capacity of all sources is bounded from above by $\Theta(W)$. Assume that all nodes are deployed in a square (the proof will carry over to the case when the deployment region is any region $\Omega$ such that $\frac{|\Omega \cap D(v, r)|}{|D(v, r)|}$ is bounded from below by some constant for any node $v \in V$ where $D(v, r)$ is a disk centered at $v$ with radius $r$ ). Let $n_{1}$ be the maximum number of nodes that can transmit simultaneously. Let $A$ be the area of the deployment region that are covered by disks $\cup_{v \in V} D(v, r)$. Let $A_{0}=\pi r^{2}$. Then obviously, $n_{1} \cdot A_{0} / 4 \leq A$ since each transmission of a node will occupy at least area $A_{0} / 4$ inside the square deployment region. This implies that the total number of bits that can be transmitted in a second all over the network is at most $W \cdot \frac{A}{A_{0} / 4}$.

On the other hand, each bit from any source node needs to be relayed by some connected dominating set. Notice that here bit from different source node may use different connected dominating set. Let us consider the area covered by any fixed instance of CDS, i.e., $\cup_{v \in C D S} D(v, r)$. Notice that here CDS denotes a fixed connected dominating set. Notice that every node $u \in V$ is either in CDS or is adjacent to a node in CDS. We can cluster nodes into $|C D S|$ clusters: each cluster $\mathcal{C}_{i}$ contain one node $v_{i}$ from the CDS and the nodes dominated by this node. Then

$$
\left|\cup_{v \in \mathcal{C}_{i}} D(v, r)\right| \leq \pi(2 r)^{2}=4 A_{0}=4|D(v, r)|
$$

Since all nodes in $\mathcal{C}_{i}$ are in disk $D(v, r)$. Here $|\mathcal{X}|$ denote the area of a region $\mathcal{X}$. Thus, the area, $\cup_{v \in C D S} D(v, r)$, covered by all nodes in any CDS is at least $A / 4$. In other words, each bit from any source node will be relayed by at least $\frac{A / 4}{A_{0}}$ nodes since each relay node covers an area at most $A_{0}$.

Combining the above analysis, we know that the aggregated broadcast capacity that can be supported is at most

$$
\left(W \cdot \frac{A}{A_{0} / 4}\right) /\left(\frac{A / 4}{A_{0}}\right)=16 W=\Theta(W)
$$


On the other hand, we can perform broadcast as before using CDS. The only modification is that we need one more time slot in a scheduling period for letting some source nodes that are not in the CDS to upload its data to its dominator in the CDS. Then the data will be broadcast to the network using the same CDS for all source nodes.

\subsection{Physical Interference Model}

We then prove that the aggregated broadcast capacity for an arbitrary network is still $\Theta(W)$ when the physical interference is used, all nodes use the same transmission power $P$, and the path-loss exponent $\alpha>2$. When a node $v_{i}$ transmits, the signal strength at a node $v_{j}$ is assumed to be $P \cdot\left\|v_{i}-v_{j}\right\|^{-\alpha}$ for a constant $\alpha>2$. The background noise is assumed to be a fixed constant $N_{0}>0$ for every receiving node. Thus, a node $v_{j}$ can successfully receive data from a node $v_{i}$ only if the SINR is at least a threshold value $\eta>0$, i.e.,

$$
\frac{P \cdot\left\|v_{i}-v_{j}\right\|^{-\alpha}}{B \cdot N_{0}+\sum_{k \neq i, k \in A(t)} P \cdot\left\|v_{k}-v_{j}\right\|^{-\alpha}} \geq \eta .
$$

Here $A(t)$ is the set of nodes that are actively transmitting at time $t$ when node $v_{i}$ is transmitting. Notice that, the received signal has strength at most $P$. Thus, we need

$$
\eta<\frac{P}{B \cdot N_{0}}
$$

In the rest of sections, to give an upperbound on the broadcast capacity, we will essentially show that we can set an artificial transmission range $r_{0}$ and an artificial interference range $R_{0}$ such that the receiving nodes of a node is within distance $r_{0}$ and a transmitting node $v_{k}$ will cause interference at a node $v_{j}$ within distance $R_{0}$ if $v_{j}$ is not its intended receiver. To give a lowerbound on the broadcast capacity, we will essentially show that we can set an artificial transmission range $r_{1}$ and an artificial interference range $R_{1}$ such that, when all simultaneously transmitting nodes are separated by a distance $R_{1}$, and the receiving nodes of a transmitting node is within distance $r_{1}$, the SINR of every receiving nodes is at least $\eta$. Combining with results in Theorem 1, it is obvious that the same asymptotic bounds for broadcast capacity with protocol interference model still hold for the networks with physical interference model.

First of all, given $P, N_{0}$ and $\eta$, the maximum distance $r_{0}$ that a node $v_{i}$ can communicate with satisfies that $P \cdot r_{0}^{-\alpha} \geq$ $\eta \cdot B N_{0}$. Thus, $r_{0}$ is at most

$$
r_{0} \leq\left(\frac{P}{B \cdot N_{0} \cdot \eta}\right)^{1 / \alpha}
$$

We then show that if a receiving node $v_{j}$ is within a distance
$R_{0}$ satisfying

$$
R_{0}<\left(\frac{\eta \cdot P}{P-\eta \cdot B \cdot N_{0}}\right)^{1 / \alpha}
$$

of a transmitting node $v_{k}$, then $v_{j}$ will be interfered by $v_{k}$ if $v_{j}$ is not the intended receiver of $v_{k}$. Notice the definition of $R_{0}$ is valid since $P-\eta \cdot B \cdot N_{0}>0$. Let $v_{i}$ be the legitimate sender for node $v_{j}$. Notice that the maximum strength of the signal from $v_{j}$ received at node $v_{j}$ is at most $P$. Thus, we have the SINR at node $v_{j}$ is at most

$$
\frac{P}{B \cdot N_{0}+P \cdot\left\|v_{k}-v_{j}\right\|^{-\alpha}} \leq \frac{P}{B \cdot N_{0}+P \cdot R_{0}^{-\alpha}}<\eta
$$

The last inequality comes from the fact that $R_{0}<$ $\left(\frac{\eta \cdot P}{P-\eta \cdot B \cdot N_{0}}\right)^{1 / \alpha}$. Consequently, the node $v_{j}$ cannot receive the data from $v_{i}$ correctly.

Secondly, we show that we can set transmission range $r_{1}$ and an interference range $R_{1}$ such that the transmissions will always be successful if the receiver $v_{j}$ is within $r_{1}$ of the sender $v_{i}$ and the receiver is not within distance $R_{1}$ of any other sender $v_{k}$ for $k \neq i$. It is easy to show that every pair of active transmitting nodes are separated by at least $R_{2}=R_{1}-r_{1}$. Then the interference at node $v_{j}$ by all other active transmitting nodes (other than $v_{i}$ ), denoted by $E$, is at most

$\sum_{i=1}^{\infty} \frac{2 \pi \cdot i \cdot R_{2}}{R_{2}} \cdot \frac{P}{\left(i \cdot R_{2}\right)^{\alpha}}=\frac{2 \pi \cdot P}{R_{2}^{\alpha}} \sum_{i=1}^{\infty} \frac{1}{i^{\alpha-1}}=\frac{2 \pi \cdot P}{R_{2}^{\alpha}} \frac{1}{\alpha-2}$.

Thus, the SINR at node $v_{j}$ is

$$
\begin{aligned}
\frac{P \cdot\left\|v_{i}-v_{j}\right\|^{-\alpha}}{B \cdot N_{0}+E} & \geq \frac{P}{\left(B \cdot N_{0}+E\right) \cdot r_{1}^{\alpha}} \\
& \geq \frac{P}{\left(B \cdot N_{0}+\frac{2 \pi \cdot P}{R_{2}^{\alpha}(\alpha-2)}\right) \cdot r_{1}^{\alpha}} \\
& \geq \eta
\end{aligned}
$$

The last inequality will be true if we require that

$$
r_{1} \leq\left(\frac{P}{\left(B \cdot N_{0}+\frac{2 \pi \cdot P}{R_{2}^{\alpha}(\alpha-2)}\right) \cdot \eta}\right)^{1 / \alpha} .
$$

The interference range $R_{1}$ is then set as $R_{1} \leftarrow R_{2}+r_{1}$. Notice that, in the above analysis, we need the bound for $r_{1}$ to be larger than 1 . Otherwise, the received power $P \cdot r_{1}^{-\alpha}$ will be larger than $P$. Recall that we require $P>B \cdot N_{0} \cdot \eta$, which implies that we can choose sufficiently large constant $R_{2}$ such that $\frac{P}{\left(B \cdot N_{0}+\frac{2 \pi \cdot P}{R_{2}^{2}(\alpha-2)}\right) \cdot \eta}>1$.

Since we found constants $r_{1}, R_{1}$ and $R_{0}$, we can construct a connected dominating set of the following communication graph: two nodes are connected if their distance is no more than $r_{1}$. Several methods can guarantee 
that for each node on the constructed CDS, it has only a constant number, say $\Delta$, of neighbors in CDS. Then consider the interference graph $H$ defined on nodes in CDS: two nodes conflict with each other if their distance is at most $R_{1}$. Then it is obvious that any node in CDS has at most $\Theta\left(\frac{R_{1}^{2}}{r_{1}^{2}} \Delta\right)$ neighbors in the conflict graph. Then we can schedule nodes in CDS to transmit in a constant time-slots (depending on $R_{1}, r_{1}$, and $\Delta$ ) such that nodes in any timeslots will not interfere with each other's transmission, under the physical interference model. Thus, the total broadcast capacity is at least $W / \Theta\left(\frac{R_{1}^{2}}{r_{1}^{2}} \Delta\right)$, where $R_{1}^{2} / r_{1}^{2} \simeq\left(\frac{4 \pi \eta}{\alpha-2}\right)^{2 / \alpha}$. Consequently, we have,

Theorem 2 Assume that the channel capacity is $W$ bits/sec. Under the physical interference mode, the aggregated broadcast capacity $\Lambda(n)$ of an arbitrary wireless network is $\Theta(W)$.

\section{Broadcast Capacity using Gaussian chan- nel}

Obviously, when fixed data rate channel is used, the study in previous section shows that the broadcast capacity for an arbitrary networks is still $\Theta(W)$, when either protocol interference model or the physical interference model is used. In this section, we will study the broadcast capacity for random networks with Gaussian channel model.

\subsection{Upper Bound of Broadcast Capacity Uing Gaussian Channel}

In broadcasting data from a source node to all nodes in the network, messages will be forwarded in a spanning tree (or a collection of spanning trees sometimes). Notice that the broadcast tree can change overtime and a node may use different transmission power and thus have different set of downstream children nodes. A node $v$ will never be able to receive messages at a rate faster than the capacity of its best incident link. Recall that, under the link rate assumption, the maximum data rate that can be received by node $v$ is always from the link $u v$ with the shortest Euclidean length.

Define the nearest neighbor graph $N N G(n)$ as follows: it contains all nodes in the network and each node $v$ is connected to its nearest neighbor $u$ (with the smallest Euclidean distance). Let $M_{n}$ be the longest edge of NNG with n nodes produced by a random point process on a 2-D unit square. It was proved in [13] that

$$
\operatorname{Pr}\left(n \pi M_{n}^{2}-\log n \leq \gamma\right)=e^{-e^{-\gamma}}
$$

In other words, with high probability, the longest edge in such an $N N G(n)$ is at least $\Theta\left(\sqrt{\frac{\log n}{n}}\right)$ with high probability. When we scale the unit square area to a square of length $a$, then the longest edge $M_{n}$ in NNG will also be scaled up by a factor $a$. It is thus natural to conclude that the longest edge of NNG of nodes produced by randomly and uniformly placing $n$ points over a square of side length $a$ is $\Theta(a \log n)$ with high probability.

Theorem 3 Let $M_{n}$ be the longest edge of the nearest neighbor graph constructed from randomly and uniformly placing $n$ nodes over a square with side length $a$. If $\lim _{n \rightarrow \infty} f(n)=+\infty$, then

$$
\begin{aligned}
& \lim _{n \rightarrow \infty} \operatorname{Pr}\left(\pi n \cdot\left(\frac{M_{n}}{a}\right)^{2}-\log n+f(n) \geq 0\right)=1 \\
& \lim _{n \rightarrow \infty} \operatorname{Pr}\left(\pi n \cdot\left(\frac{M_{n}}{a}\right)^{2}-\log n-f(n) \leq 0\right)=1
\end{aligned}
$$

For example, we can set $f(n)=\log n / 2$. Then it is easy to show that $\lim _{n \rightarrow \infty} \operatorname{Pr}\left(M_{n} \geq a \sqrt{\frac{\log n}{2 \pi n}}\right)=1$, $\lim _{n \rightarrow \infty} \operatorname{Pr}\left(M_{n} \leq a \sqrt{\frac{3 \log n}{2 \pi n}}\right)=1$. Assume that $u v$ is the longest edge in NNG, i.e., $\|u v\|=M_{n}$. To improve the data rate received by a node $v$, we clearly need to reduce the interference, i.e., only one node is sending and one node $v$ is receiving. The data rate that node $v$ can receive is bounded from above by

$$
B \cdot \log \left(1+\frac{P_{\max } / M_{n}^{\alpha}}{B \cdot N_{0}}\right) .
$$

There are three different cases here. The first case is that $\lim _{n \rightarrow \infty} M_{n}=\infty$, i.e., $\lim _{n \rightarrow \infty} a \sqrt{\frac{\log n}{n}}=\infty$. In this case, the broadcast capacity, with high probability, is at most

$$
\begin{aligned}
B \cdot \log \left(1+\frac{P_{\max } /\left(a^{2} \frac{\log n}{2 \pi n}\right)^{\alpha / 2}}{B \cdot N_{0}}\right) & \simeq B \cdot \frac{P_{\max } \cdot a^{\alpha}\left(\frac{\log n}{2 \pi n}\right)^{-\frac{\alpha}{2}}}{B \cdot N_{0}} \\
& =\frac{P_{\max }}{N_{0} \cdot\left(a \cdot \sqrt{\frac{\log n}{2 \pi n}}\right)^{\alpha}}
\end{aligned}
$$

This first approximation comes from the fact that $\log (1+$ $x) \simeq x$ when $x \rightarrow 0$ and $\lim _{n \rightarrow \infty} a \sqrt{\frac{\log n}{n}}=\infty$. This concludes that the maximum broadcast data rate that can be supported by a random extended network is at most $\frac{P_{\max }}{N_{0} \cdot\left(a \cdot \sqrt{\frac{\log n}{2 \pi n}}\right)^{\alpha}}$ with high probability. This implies that for a network of $n$ nodes deployed in a square region with sidelength $a$, the larger the region, the smaller the broadcast capacity upperbound that can be achieved.

Theorem 4 When $\lim _{n \rightarrow \infty} a \sqrt{\frac{\log n}{2 \pi n}}=\infty$, the broadcast capacity is at most $\frac{P_{\max }}{N_{0} \cdot\left(a \cdot \sqrt{\frac{\log n}{2 \pi n}}\right)^{\alpha}}$ with high probability. 
The second case is that $\lim _{n \rightarrow \infty} M_{n}=\Theta(1)$, and the third case is that $\lim _{n \rightarrow \infty} M_{n}=0$. In these two cases, the power attenuation model $P / d^{\alpha}$ may not applicable when $d<1$ : the received power is larger than the sending power! To address this issue, we assume that the power received at a distance $d$ from receiver is $P /\left(1+d^{\alpha}\right)$. Under this new power attenuation model, it is easy to show that when $\lim _{n \rightarrow \infty} M_{n}$ is at most some constant $c$, then the link capacity over longest edge in NNG is at most $B \log \left(1+\frac{P_{\max }}{B \cdot N_{0} \cdot\left(1+c^{\alpha}\right)}\right) \leq B \log \left(1+\frac{P_{\max }}{B \cdot N_{0}}\right)$, which is a constant.

Theorem 5 When $\lim _{n \rightarrow \infty} a \sqrt{\frac{\log n}{2 \pi n}}=c=O(1)$, the broadcast capacity is at most $B \log \left(1+\frac{P_{\max }}{B \cdot N_{0} \cdot\left(1+c^{\alpha}\right)}\right)$ with high probability.

Notice that for a random network, the transmission power should be the minimum to guarantee that the network is connected with high probability. In our previous studies, we assume that the Gaussian channel capacity is $B \log (1+S I N R)$ no matter how small SINR could be. In practice, all wireless devices have certain lower bound on the minimum SINR $\eta$. If this is the case, then we know that the maximum distance a node can receive signal correctly is at most $r_{0} \leq\left(\frac{P_{\max }}{B \cdot N_{0} \cdot \eta}\right)^{1 / \alpha}$. Thus, the longest edge in the Euclidean minimum spanning tree (which has the same asymptotic value as that of the NNG) should be no more than $r_{0}$ with high probability. Observe that when $r_{0}$ is bounded from above by a constant, we know that the largest value for side-length $a$ satisfying

$$
a \leq r_{0} \cdot \sqrt{\frac{2 \pi n}{\log n+f(n)}}
$$

for a certain function $f(n) \rightarrow \infty$. When $a=r_{0}$. $\sqrt{\frac{2 \pi n}{\log n+f(n)}}$, the network will be connected with probability $e^{-e^{-f(n)}}$ when $n \rightarrow \infty$. This consequently implies that the broadcast capacity is at least a constant $B \log (1+\eta)$ and at most a constant $B \log \left(1+\frac{P_{\max }}{B \cdot N_{0}}\right)$.

\subsection{Lower Bound of Broadcast Capacity Uing Gaussian Channel}

We then use a constructive method to show that such broadcast capacity can be achieved with high probability for a random network. The basic idea of this constructive method again relies on constructing a good connected dominating set: each node in the CDS only has a constant number of interfering nodes in the CDS. Thus, we can find a schedule with a constant period, say $T$, such that each node in the CDS will have at least one time-slot to send its data in
$T$. Another important observation is that the smallest transmission radius needed to have a connected network is also asymptotically $M_{n} \simeq a \cdot \sqrt{\frac{\log n}{2 \pi n}}$. This implies that the continuous data rate that can be supported by each node in the $\mathrm{CDS}$ is also in the order of $B \log \left(1+\frac{P_{\max }}{B \cdot N_{0} \cdot M_{n}^{\alpha}}\right)$. Combining the fact that each node in CDS can use at least one time slot to send data every constant $T$ slots, the achieved broadcast capacity is still in the order of $B \log \left(1+\frac{P_{\max }}{B \cdot N_{0} \cdot M_{n}^{\alpha}}\right) / T$.

In [17], the author did not specifically use the CDS structure. It partitions the deployment region into cells and will select one node from each cell as relay node for broadcast, i.e., these representative nodes will be in CDS. The sidelength $\ell$ of the cell should be carefully selected. Recall that the channel model is assumed to be Gaussian channel. Thus, under a schedule of transmissions, we need to show that the actual data rate supported by a node is indeed $\Theta\left(B \log \left(1+\frac{P_{\max }}{B \cdot N_{0} \cdot M_{n}^{\alpha}}\right) / T\right)$.

Theorem 6 Assume that $\alpha>2$. For any given $r>0$, under the Gaussian channel and the general physical interference model, there exists a TDMA scheduling, where one node per square of edge length $\ell$ can transmit to nodes located within a radius $r$, with fixed rate $R(r, \ell)$ given as

$$
R(r, \ell) \geq B \log \left(1+\frac{P_{\max }}{r^{\alpha} \cdot\left(B N_{0}+P_{\max } \cdot K / \ell^{\alpha}\right)}\right)
$$

where $K \leq \frac{2 \pi}{\alpha-2}$ is a constant.

Notice that here $K=2 \pi \sum_{i=1}^{\infty} \frac{1}{i^{\alpha-1}}$, which converges to a constant $\frac{2 \pi}{\alpha-2}$ when $\alpha>2$. When $\alpha=2, K$ is not a constant anymore: it is $O(\log n)$ instead. The theorem directly follows from the fact that the overall interference $E$ from all transmitting nodes that are not in the same cell is at most $K \cdot P_{\max } / \ell^{\alpha}$ and the channel capacity is at least $B \log \left(1+\frac{P_{\max } \cdot r^{-\alpha}}{B N_{0}+E}\right)$. Based on this theorem, we know that the broadcast capacity is at least $R(r, \ell) / T$, where $T \leq \frac{\ell^{2}}{r^{2}}$ is the time-slots needed to schedule all nodes. When we select $r=M_{n} \simeq a \sqrt{\frac{\log n}{2 \pi n}}$, and $\ell=C \cdot r$ for a constant $C>1$, we have the following theorem

Theorem 7 When $\alpha>2, \lim _{n \rightarrow \infty} M_{n}=\infty$, i.e., $\lim _{n \rightarrow \infty} a \sqrt{\frac{\log n}{2 \pi n}}=\infty$, and select $r=M_{n}$ and $\ell=C \cdot r$, the broadcast capacity achieved by our scheme is at least

$$
\frac{P_{\max }}{C \cdot N_{0} \cdot\left(a \sqrt{\frac{\log n}{2 \pi n}}\right)^{\alpha}}
$$

The theorem directly follows from Theorem 7 and $\log (1+x) \geq x / 2$ for $x \rightarrow 0$. For example, when $a=\sqrt{n}$, then $\ell=\Theta(r(n))=\Theta(\sqrt{\log n})$. Theorem 7 implies that, the achievable data rate by nodes in the CDS (representative nodes selected from all cells) is in the order of 
$\Theta\left(\frac{P_{\max }}{\ell^{\alpha}}\right)=\Theta\left(\frac{P_{\max }}{(\log n)^{\frac{\alpha}{2}}}\right)$. When the node deployment $a$ satisfies that $a \sqrt{\frac{\log n}{n}}=O(1)$, the broadcast capacity is actually $\Theta(1)$ for $\alpha>2$. Thus, we have the following theorem.

Theorem 8 Assume that capacity-achieving Gaussian channel codes are used and $\alpha>2$. When $\lim _{n \rightarrow \infty} a \sqrt{\frac{\log n}{2 \pi n}} \leq b$ for a constant $b$, and select $r=M_{n}$ and $\ell=C \cdot r$ for a constant $C>1$, the broadcast capacity achieved by our scheme is at least $B \cdot \log (1+$ $\left.\frac{P_{\max }}{B \cdot N_{0} b^{\alpha}+K \cdot C^{-\alpha} P_{\max }}\right) / C^{2}=\Theta(1)$.

Observe that all the above analysis will carry over to the dense model. However, for the dense model, the power attenuation model $\frac{P}{\|u-v\|^{\alpha}}$ will result in a non-valid scenario where the receiving power is larger than the sending power. To remedy this, we use the following power attenuation model $\frac{P}{(1+\|u-v\|)^{\alpha}}$.

\section{Multicast Capacity}

In previous sections, we studied the broadcast capacity of various networks under various interference models. In this section, we study the multicast capacity of a random networks under the physical interference model. Assume that $n$ wireless nodes are randomly deployed in a square region with side-length $a$. We further assume that each wireless node can transmit/receive at $W$ bits/second over a common wireless channel. For each node $v_{i}$, we randomly pick $k-1$ nodes from the other $n-1$ nodes as the receivers of the multicast session rooted at node $v_{i}$. The aggregated multicast capacity is defined as the total data rate of all multicast sessions in the network. Li et al. [12] studied multicast capacity for large scale wireless ad hoc networks under the Protocol Interference model: all nodes have the uniform transmission range $r$ and uniform interference range $R>r$.

Recall that in Section 3.2, we showed that, under the physical interference model, we can define transmission range $r_{0}$, and interference range $R_{0}$ such that

1. the maximum distance that a node $v_{i}$ can communicate with is at most $r_{0}$

2. if a receiving node $v_{j}$ is within a distance $R_{0}$ of a transmitting node $v_{k}$, then $v_{j}$ will be interfered by $v_{k}$ if $v_{j}$ is not the intended receiver of $v_{k}$.

We also can define another range $r_{1}$ and interference range $R_{1}$ such that

1. the transmissions will be successful if the receiver $v_{j}$ is within $r_{1}$ of the sender $v_{i}$, and, the receiver is not within distance $R_{1}$ of any other sender $v_{k}$ for $k \neq i$.

When we multicast from one source node $v_{i}$ to all its $k-1$ receivers $U_{i}$, it is more likely that other nodes will also get a copy of the data. Here, for the purpose of analysis, when a node $v$ sends data to one of its "neighboring" nodes, all its neighboring nodes will be charged a copy of the data. Notice that here a node $w$ may not be the intended receiver of $v$. However, since when $v$ is transmitting, any node $w$, that is within distance $R_{0}$ of $v$, cannot receive data simultaneously from any other transmitting node due to physical interference, we will say that node $w$ also gets a copy of the data. For multicast with $k-1$ receivers, clearly, at least $k$ nodes will get a copy of the data. Generally, assume that $C_{i}$ nodes will get a copy of the data when the $k-1$ receivers are randomly selected for each possible source node $v_{i}$. Obviously, with a set $\mathcal{S}$ of source nodes, $\sum_{v_{i} \in \mathcal{S}} \lambda_{i} \cdot C_{i} \leq n \cdot W$, where source node $v_{i}$ generates data at rate $\lambda_{i}$. Further assume that $\operatorname{Pr}\left(C_{i} \geq C\right) \rightarrow 1$ as $n$ or $k$ goes to infinity. Then the total multicast capacity satisfies, almost surely,

$$
\Lambda_{k}(n)=\sum_{v_{i} \in \mathcal{S}} \lambda_{i} \leq \frac{n \cdot W}{C} .
$$

For any multicast tree $T$, let $D(T)$ denote the region covered by the set of disks centered at every internal node of $T$ with a radius $r$. The following were proved in [12].

Lemma 9 The area of $D(T)$, denoted by $|D(T)|$ is at most $\tau \sqrt{k} a \cdot 2 r+k \cdot \pi r^{2} / 2$ and w.h.p.is at least $\frac{\tau \sqrt{k} a \cdot r}{c_{0}}$ when $k<$ $\left(\frac{\tau(1-(6(d+1) \cdot \rho))}{6(d+1)+1}\right)^{2} \cdot \frac{a^{2}}{r^{2}}$, for some constant $c_{0}=1 /(4 \rho \pi)$, where $0<\rho<\frac{1}{12(d+1)}$ and constant $d \leq 13$.

Here $\tau$ is some constant independent of $n, k, r$ and $a$.

Lemma 10 With high probability, the number $C$ of nodes that get a copy of the multicast data satisfies $C>\frac{\tau \cdot r \cdot \sqrt{k} \cdot n}{2 c_{0} a}$.

Consequently, we know that the number of nodes that will get a copy of the multicast data under physical interference model is at least $\frac{\tau \cdot R_{0} \cdot \sqrt{k} \cdot n}{2 c_{0} a}$,w.h.p.. This implies that the aggregated multicast capacity is at most, w.h.p.,

$$
\frac{n W}{\frac{\tau \cdot R_{0} \cdot \sqrt{k} \cdot n}{2 c_{0} a}}=\frac{2 c_{0}}{\tau} \cdot \frac{a}{R_{0}} \cdot \frac{W}{\sqrt{k}}
$$

Notice that to guarantee a connected network, we need that the maximum transmission distance $r_{0}$ should satisfy that $r_{0}>a \sqrt{\frac{\log n}{\pi n}}$. Notice that $R_{0}=r_{0} \cdot\left(\frac{B \eta^{2} N_{0}}{P-B \eta N_{0}}\right)^{1 / \alpha}$. Notice that Lemma 10 is valid only if the number of receivers is at most $O\left(a^{2} / r_{0}^{2}\right)$. Thus, we have

Theorem 11 When $k=O(n / \log n)$, the aggregated multicast capacity is at most, w.h.p.,

$$
\frac{n W}{\frac{\tau \cdot R_{0} \cdot \sqrt{k} \cdot n}{2 c_{0} a}}=\frac{2 c_{0}}{\tau} \cdot \frac{a}{R_{0}} \cdot \frac{W}{\sqrt{k}}=O\left(\sqrt{\frac{n}{k \log n}} \cdot W\right) .
$$

We then study a lower bound on the aggregated multicast capacity when $k=O(n / \log n)$. In [12], a routing 
scheme based on a connected dominating set (CDS) was proposed to achieve asymptotic optimum multicast capacity for protocol interference model. First, we construct a CDS using squarelet partition method. Then given a set $U_{i}$ of multicast receivers and source node, we build an Euclidean minimum spanning tree $\left(\operatorname{EMST}\left(U_{i}\right)\right)$ spanning these nodes. Then for each edge $u v$ in $\operatorname{EMST}\left(U_{i}\right)$, we use the shortest hop path in CDS to connect them. The resulting structure, composed of all shortest paths for all edges in EMST, is the final routing structure $M T\left(U_{i}\right)$. Here we show that that routing scheme also achieves asymptotic optimum multicast capacity for physical interference model. In our routing scheme, when we build CDS, we use radius $r_{1}$ as the transmission radius, i.e., two nodes can communicate directly only if their Euclidean distance is at most $r_{1}$. To schedule transmissions using TDMA, two nodes within distance $R_{1}$ will not be scheduled simultaneously for transmitting data. In other words, $r_{1}$ is the logic transmission range and $R_{1}$ is the logic interference range to mimic the proofs used for protocol interference model.

First of all, it was proved in [12] that the total Euclidean length of edges in $M T\left(U_{i}\right)$ is within a small constant factor of $\operatorname{EMST}\left(U_{i}\right)$, which is at most $2 \sqrt{2} \sqrt{k} \cdot a$. Thus, the total area covered by all nodes within radius $R_{1}$ will be at most $2 \sqrt{2} \sqrt{k} \cdot a \cdot\left(2 R_{1}\right)$. Thus, the expected number $E(c)$ of nodes that will get a "copy" of the multicast data is at most $2 \sqrt{2} \sqrt{k} \cdot a \cdot\left(2 R_{1}\right) \cdot \frac{n}{a^{2}}$. Then we can show that $C$ is at most $2 E(c)$ with high probability. Consequently, we have

Theorem 12 When $k=O(n / \log n)$, the aggregated multicast capacity is at least, w.h.p.,

$$
\frac{n W}{2 E(C)}=\frac{n W}{2 \sqrt{2} \sqrt{k} \cdot a \cdot\left(2 R_{1}\right) \cdot \frac{2 n}{a^{2}}}=\Omega\left(\sqrt{\frac{n}{k \log n}} \cdot W\right) .
$$

This is due to the fact that $R_{1}=\Theta(1)$, and we can set $a=\Theta\left(\sqrt{\frac{n}{\log n}}\right)$ to have a connected network with high probability.

When $k=\Omega(n / \log n)$, we can show that multicast is asymptotically same as broadcast, and thus, the asymptotic aggregated multicast capacity is in the order of $\Theta(W)$. The proof details are omitted here due to space limit.

\section{Literature Reviews}

Gupta and Kumar [5] studied the asymptotic capacity of a multi-hop wireless networks for two different models. When each wireless node is capable of transmitting at $W$ bits per second using a fixed range, the throughput obtainable by each node for a randomly chosen destination is $\Theta\left(\frac{W}{\sqrt{n \log n}}\right)$ bits per second under a non-interference protocol, where $n$ in number of nodes. Similar results also hold for physical interference model. Grossglauser and Tse [4] recently showed that mobility actually can help to improve the capacity if we allow arbitrary large delay. Their main result shows that the average long-term throughput per source-destination pair can be kept constant even as the number of nodes per unit area increases. The main idea used in [4] is to use some intermediate node to serve as ferry node: this node will carry the data from the source node and move around and it will dump the data to the target node when it is within its communication range. In summary, for random networks, under the protocol model, the achievable throughput capacity $\lambda(n)$ and the average travel distance $\bar{L}$ satisfies $\lambda(n) \cdot \bar{L} \leq \Theta\left(\frac{W}{\Delta^{2} n \cdot r(n)}\right)$. This phenomenon has also been observed in [11]. In [3] Gastpar and Vetterli studied the capacity of wireless networks when network coding can be used to improve the capacity.

Capacity can also be generalized to the notion of capacity region. For a given statistical description of the network, a set of constraints (such as power per node, link capacity, etc.), and a list of desired communication pairs, the capacity region is the closure of all rate tuples that can be achieved simultaneously. Here a rate tuple specifies the rate for each of the desired communications. Kyasanur and Vaidya [10] studied the capacity region on random multi-hop multiradio multi-channel wireless networks when there are total $c$ channels available and each node has $m \leq c$ wireless interfaces. On the other aspect, several papers $[2,9] \mathrm{re-}$ cently studied how to satisfy a certain traffic demand vector from all wireless nodes by a joint routing, link scheduling, and channel assignment under certain wireless interference models.

Broadcast capacity of an arbitrary network has been studied in $[8,15]$. They essentially show that the broadcast capacity of a given network is $\Theta(W)$ for single source broadcast and the achievable broadcast capacity per node is only $\Theta(W / n)$ if each of the $n$ nodes will serve as source node. They assume a simple channel model: when no interference exists, a node can transmit to its neighbors at data rate at most $W$ bits/second. They also assume that all wireless nodes have a uniform transmission range $r$ and uniform interference range $R=\Theta(r)$ : a node $v$ cannot receive data from a transmitting node $u$ with $\|u-v\| \leq r$ if there is another transmitting node $w$ with $\|w-v\| \leq R$. Keshavarz-Haddad et al. [7] studied the broadcast capacity with dynamic power adjustment for physical interference model. Their results are most similar to ours. They mainly considered the dense model. They did consider both physical interference model and Generalized Physical Interference model (called Gaussian channel model here). In physical model used, a node can receive data correctly only if the SINR is at least a threshold. The Physical Model models interference more accurately, but still assigns a constant transmission rate once successful transmission is guaran- 
teed. The Generalized Physical Model allows for a transmission rate that depends on the level of interference and the distance between sender and receiver and thus allows for a more precise assessment of the broadcast capacity. In this model the transmission rate $W_{i, j}$ between a sender $i$ and a receiver $j$ is determined using Shannon's formula for a wireless channel with additive Gaussian white noise. Li et al. [12] studied multicast capacity for large scale wireless ad hoc networks under the Protocol Interference model.

\section{Conclusions}

In this paper, we essentially studied the broadcast capacity that can be achieved by some wireless networks. We derive analytical upper bounds and lower bounds on broadcast capacity of a wireless network when all nodes in the network has the same bounded transmission power $P$ and nodes are deployed in a square of side-length $a$. We also generalize our results to multicast capacity. Assume that for each node $v$ in the network, we randomly select $1 \leq k<n$ nodes as receiver nodes of a multicast from $v$. We show that the asymptotic aggregated multicast capacity is $\Theta\left(\sqrt{\frac{n}{k \log n}} W\right)$ when $k=O(n / \log n)$ and the aggregated multicast capacity is $\Theta(W)$ otherwise. It is interesting to study the broadcast capacity and the multicast capacity when nodes could dynamically adjust its coding to change the data rate based on the receivers.

\section{References}

[1] Ahn, G.-S., Miluzzo, E., Campbell, A. T., Hong, S. G., AND CuOMO, F. Funneling-mac: A localized, sinkoriented mac for boosting fidelity in sensor networks. In ACM SenSys (2006).

[2] Alicherry, M., Bhatia, R., And Li, L. E. Joint channel assignment and routing for throughput optimization in multiradio wireless mesh networks. In MobiCom (2005), pp. 5872.

[3] Gastpar, M., and Vetterli, M. On the capacity of wireless networks: the relay case. In IEEE INFOCOM (2002).

[4] Grossglauser, M., And Tse, D. Mobility increases the capacity of ad-hoc wireless networks. In INFOCOMM (2001), vol. 3, pp. $1360-1369$.

[5] GuPta, P., AND Kumar, P. Capacity of wireless networks. Tech. rep., University of Illinois, Urbana-Champaign, 1999.

[6] Gupta, P., AND Kumar, P. R. Critical power for asymptotic connectivity in wireless networks. Stochastic Analysis, Control, Optimization and Applications: A Volume in Honor of W.H. Fleming, W. M. McEneaney, G. Yin, and Q. Zhang (Eds.) (1998).
[7] Keshavarz-Haddad, A. And Riedi R. On the Broadcast Capacity of Multihop Wireless Networks: Interplay of Power, Density and Interference, 4th IEEE SECON, June 2007. $x$

[8] Keshavarz-Haddad, A., Ribeiro, V., And Riedi, R. Broadcast capacity in multihop wireless networks. In MobiCom (2006), ACM Press, pp. 239-250.

[9] Kodialam, M., And Nandagopal, T. Characterizing achievable rates in multi-hop wireless networks: the joint routing and scheduling problem. In MobiCom (2003), pp. 42-54.

[10] Kyasanur, P., AND VAidya, N. H. Capacity of multichannel wireless networks: impact of number of channels and interfaces. In MobiCom (2005), pp. 43-57.

[11] Li, J., Blake, C., Couto, D. S. J. D., Lee, H. I., AND MORRIS, R. Capacity of ad hoc wireless networks. In ACM MobiCom (2001).

[12] Li, X.-Y., TANG, S.-J. ANd Frieder, O. Multicast Capacity of Large Scale Ad Hoc Wireless Networks. In ACM MobiCom (2007).

[13] Penrose, M. A strong law for the largest nearest neighbor link between random points. Journal of London Mathematical Society 60 (1999), 951-960.

[14] Santi, P., And Blough, D. M. The critical transmitting range for connectivity in sparse wireless ad hoc networks. IEEE Trans. on Mobile Computing 2 (Mar. 2003), 25-39.

[15] TAVLI, B. Broadcast capacity of wireless networks. IEEE Communication Letters 10, 2 (February 2006).

[16] Xue, F., And Kumar, P. R. The number of neighbors needed for connectivity of wireless networks. 